\title{
Revisión sistemática de vacuna cuadrivalente vs nonavalente como tratamiento profiláctico para cáncer cervicouterino causado por el virus del papiloma humano (MPH)
}

Becerra-Zúñiga Patricia*, Enríquez-Gómez Shaden*, Ruiz-Rocha Laura Fernanda*, Palacios-Méndez Pedro Héctor*, López-Rodríguez Diego*, Arias-Ulloa Raúl**, Terrones-Saldívar Ma. del Carmen**

\section{Resumen}

- Introducción: se considera que la vacunación profiláctica contra el virus - del papiloma humano (VPH) es la intervención más efectiva para dis-

- minuir la prevalencia del cáncer cérvico-uterino; sin embargo, a pesar de

- la implementación de programas de vacunación, se han identificado ba-

- rreras relacionadas con la seguridad y los efectos adversos, motivo por el

- cual existe una baja cobertura de ésta. Objetivo: analizar y sintetizar la

- evidencia disponible acerca de la efectividad de la vacuna cuadrivalente

- y la nonavalente como método profiláctico para disminuir la incidencia

- de CaCu. Material y métodos: se realizó una revisión electrónica de

- artículos en los que se analizaba la eficacia de cada tipo de vacuna

- en la población de interés, así como estudios comparativos de ambos

- tipos de vacuna. Resultados: en cuanto a la eficacia de las vacunas, la

- evidencia científica reporta que, después de la vacunación, existe la

- formación de anticuerpos contra los diferentes tipos de VPH. También

- se reporta la persistencia de anticuerpos (evaluado en cada estudio) y

- por cuánto tiempo se encontró efectividad de la vacuna. En cuanto a

- la seguridad, se encontró que los efectos locales y sistémicos son más

- comunes tras la aplicación de la vacuna nonavalente. Conclusiones: los

- ensayos cli?nicos aleatorizados analizados permiten establecer el marco

- de seguridad, eficacia e inmunogenicidad de las vacunas tetravalen-

- te y nonavalente para el VPH. Además, han demostrado que ambas

- vacunas son altamente eficaces en la prevención de la infección por

- VPH 16 y 18; adicionalmente, la nonavalente muestra eficacia clínica

- contra los tipos 31, 33, 45, 52 y 58. Se debe reforzar la cobertura de

- la vacunación en la población blanco. LUX MÉDICA AÑO 14, NÚMERO 42, SEPT-DIC 2019, PP 37-48.

Palabras clave: virus del papiloma humano, VPH, vacuna tetravalente, vacuna cuadrivalente, vacuna nonavalente.

\section{Abstract}

Introduction: Prophylactic vaccines against human papillomavirus (HPV) are considered the most effective intervention for the control of the burden of HPV-related disease. Objective: To analyze and determine if the nonavalent vaccine compared to the quadrivalent vaccine is more effective as a prophylactic method to reduce the incidence of cervical cancer caused by human papillomavirus (HPV). Methods: An electronic review of articles in which the efficacy of each type of vaccine in the population of interest was analyzed, as well as comparative studies of both types of vaccine. Results: The effectiveness of the studies was assessed according to the prevalence of cases of the different types of HPV after the application of the vaccine, immunogenicity demonstrates the creation of antibodies for the different types of HPV. On the other hand, the persistence of antibodies shows if the studies assessed their presence after a specific time (evaluated in each study) and for how long the vaccine's effectiveness was found. Conclusions: The results analyzed from randomized clinical trials have shown that both vaccines are highly effective in preventing the types of high-risk HPVs included in the vaccine (HPVs 16 and 18). Additionally, nonavalent shows clinical efficacy against types 31, 33, 45, 52, and 58. LUX MÉDICA AÑO 14, NÚMERO 42, SEPT-DIC 2019, PP 37-48.

* Estudiantes del octavo semestre de la carrera de Medicina del Centro de Ciencias de la Salud de la Universidad Autónoma de Aguascalientes.

** Profesor de Tiempo Completo, Investigador del Departamento de Salud Pública del Centro de Ciencias de la Salud de la Universidad Autónoma de Aquascalientes. Correo electrónico rarias@correo.uaa.mx

*** Profesora de Tiempo Completo, Investigadora del Departamento de Medicina del Centro de Ciencias de la Salud de la Universidad Autónoma de Aguascalientes. Correo electrónico mcterron@correo.uaa.mx

Fecha de recibido: 1 de marzo 2019

Fecha de aceptación: 1 de agosto 2019

Correspondencia: Dra en C Ma del Carmen Terrones Saldívar Edificio 101, planta alta Campus universitario. Avenida Universidad 940. Código postal 20131. Aguascalientes, Ags., México. Teléfono 4499108443 Correo electrónico mcterron@correo.uaa.mx 


\section{Introducción}

Los virus del papiloma humano (VPH) son un grupo de virus de ADN de doble banda que pertenecen a la familia Papovaviridae, no poseen envoltura, y tienen un diámetro aproximado de $52-55 \mathrm{~nm}$. Las partículas virales están compuestas por una cápside proteica, conformada en un $95 \%$ por la proteína L 1 y en un $5 \%$ por la proteína L2, las cuales se ensamblan para formar capsómeras heicosaédricas y que son usadas para la fabricación de vacunas profilácticas (Figura 1). ${ }^{1,2}$

Hacia el interior de la cápside se encuentra un DNA circular de doble cadena de aproximadamente 8000 pares de bases, constituido por ocho genes y una región regulatoria no codificante, la cual contiene sitios de unión para factores proteicos y hormonales del hospedero, necesarios para que el virus pueda completar su ciclo de replicación. ${ }^{1}$

En México, el cáncer cervicouterino ocupa el segundo lugar entre las neoplasias en la mujer y constituye uno de los principales problemas de salud pública. La infección por el virus del papiloma humano (VPH) ha suscitado gran interés en las últimas décadas debido a su relación causal con el cáncer cervico-uterino. ${ }^{1}$

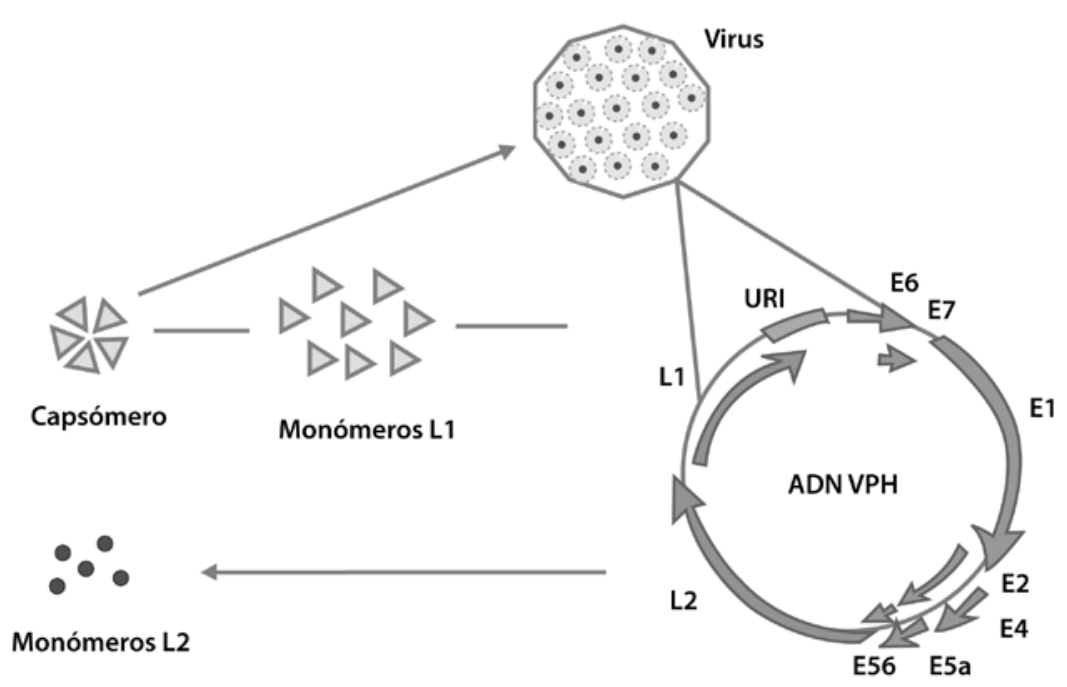

Figura 1. Estructura del virus del papiloma humano (VPH)2. Adaptado de New prphylactics human papilloma virus (HPV) vaccines against cervical cancer por Barra Fet al, 2008, Journal of Obstetrics and Gynaecology, https://doi.org/10.1080/01443615.2018.1493441.

Actualmente, existen tres vacunas profilácticas aceptadas para la prevención de enfermedades causadas por VPH. La primera que obtuvo la autorización fue la cuadrivalente, contra los virus del papiloma virus (VPH) 6, 11, 16 y 18 en 2006; la bivalente, contra los 
VPH 16 y 18, fue autorizada en 2007 y la nonavalente (contra VPHs 6, 11, 16, 18, $31,33,45,52$ y 58) en $2014 .^{2}$

En la tabla 1 se muestran detalles del esquema de vacunación. Estas vacunas profilácticas contra el VPH utilizan la proteína L1 como antígeno. Cuando la proteína L1 es expresada en sistemas in vitro, tiene la capacidad de autoensamblarse espontáneamente, adquiriendo una conformación estructural y antigénica similar al virus nativo, pero sin ser infecciosa ni cancerígena, ya que no contiene el ácido desoxirribonucleico (ADN) viral. Tales estructuras se conocen con el nombre de partículas similares al virus (VLP por sus siglas en inglés, virus like particles), que son mediadas por la producción de anticuerpos neutralizantes que reconocen epítopes específicos del genotipo viral. ${ }^{3}$

\section{Tabla |}

Esquema de vacunación de las vacunas cuadrivalente y nonavalente.

\begin{tabular}{|l|l|l|}
\hline Esquema de vacunación & $\begin{array}{l}9-13 \text { años: } 2 \text { dosis a los } 0,6 \text { meses. } \\
\text { Mayores } 13 \text { años: } 3 \text { dosis a los } 0,2 \\
\text { y } 6 \text { meses. }\end{array}$ & $\begin{array}{l}9-26 \text { años: } 3 \text { dosis a los } 0,2 \text { y } 6 \\
\text { meses. }\end{array}$ \\
\hline Vía de administración & Intramuscular; músculo deltoides. & Intramuscular; músculo deltoides. \\
\hline Nombre comercial & Gardasil@. & Gardasil 9@. \\
\hline
\end{tabular}

Además, se ha identificado la preocupación en la población acerca de la seguridad de la vacuna y los eventos adversos relacionados (EA) como una barrera que ha limitado la cobertura de la vacunación contra el VPH. El efecto adverso más común reportado tanto para las vacunas en los ensayos como para la experiencia clínica es la reacción en el sitio de la inyección, particularmente descrita como dolor, edema y eritema en el $95 \%$ de los casos de intensidad leve-moderada. ${ }^{3}$

El presente trabajo tiene como objetivo analizar la información relevante disponi- ble en bases de datos acerca de la efectividad para disminuir la incidencia del cáncer cervicouterino causado por el virus VPH, de la vacuna cuadrivalente vs la nonavalente. Se reporta de acuerdo con la declaración PRISMA (Preferred Reporting Items for Systematic Reviews and Meta-analyses), basándonos en la siguiente pregunta (tabla 2): en mujeres en edad reproductiva susceptibles a VPH, ¿la efectividad, inmunogenicidad y seguridad es mejor con la vacuna nonavalente o con la vacuna tetravalente?

\section{Tabla 2}

Esquema de la pregunta PICO.

\begin{tabular}{|l|l|l|l|}
\hline P & I & C & 0 \\
\hline $\begin{array}{l}\text { Mujeres en edad repro- } \\
\text { ductiva susceptibles a } \\
\text { VPH. }\end{array}$ & Vacuna nonavalente. & Vacuna tetravalente. & $\begin{array}{l}\text { Efectividad, inmunogeni- } \\
\text { cidad, seguridad. }\end{array}$ \\
\hline
\end{tabular}




\section{Metodología}

\section{Base de datos y estrategia de búsqueda}

Se realizó la búsqueda en las siguientes bases de datos: PubMed, EBSCO, Oxford, Science Direct, Elsevier y Google Scholar, en las que se encontraron 42 artículos, de los cuales sólo 16 cumplieron con los criterios de inclusión. No se aplicaron restricciones respecto al periodo de tiempo de evaluación; sin embargo, se limitó la búsqueda en la literatura a artículos en los idiomas inglés y español.

Se realizó una revisión electrónica de artículos que incluían ensayos clínicos aleatorizados doble ciego controlado, estudio VIVIANE, estudio PATRICIA, ensayos clínicos aleatorizados doble ciego placebo controlado, los cuales evaluaban la eficacia de cada tipo de vacuna en la población de interés, así como estudios comparativos entre la vacuna tetravalente y nonavalente.

Se utilizó una combinación de palabras claves, marcadores Booleanos y limitadores para obtener una serie de artículos pertinentes para la presente revisión. En la Tabla 3 se muestra la metodología utilizada según la base de datos.

Además, se realizó una selección de documentos mediante la técnica metodológica denominada "bola de nieve" o "Snowball", con la se recuperaron documentos desde las referencias encontradas en otros.

\section{Extracción de datos y síntesis}

Se revisaron los artículos de forma independiente y se realizó una evaluación y síntesis de los mismos para su posterior comparación en conjunto.

\section{Criterios de inclusión y exclusión}

Inclusión:

- Estudios que incluyeran la eficacia, inmunogenicidad y/o seguridad de la vacuna nonavalente $\mathrm{y} / \mathrm{o}$ tetravalente contra el VPH.

- Estudios en los que se evaluaran sólo mujeres o que compararan hombres y mujeres.

- Estudios que se hayan publicado en español o inglés.

- Estudios que compararan un solo tipo de vacuna contra control o placebo.

- Estudios clínicos aleatorizados.

Exclusión:

- Estudios que sólo evaluaran hombres.

- Estudios que compararan la vacuna bivalente con la tetravalente.

- Estudios que no evaluaran eficacia, inmunogenicidad y/o seguridad de la vacuna nonavalente $\mathrm{y} / \mathrm{o}$ tetravalente contra el VPH.

- Revisiones de literatura y/o sistemáticas.

- Estudios que se hayan publicado en otro idioma que no fuera inglés o español.

De la búsqueda en las bases de datos se encontraron 42 artículos, cuatro de ellos duplicados, por lo que, al ser eliminados, quedaron 38 artículos que fueron examinados para posteriormente descartar 10 de ellos con los criterios de exclusión. Se concluyó la selección de los artículos con la lectura completa de los mismos y excluyendo 12 artículos debido a discrepancias en los métodos, resultados o del estudio en general. Finalmente, se realizó la revisión sistemática con los 16 artículos restantes. 


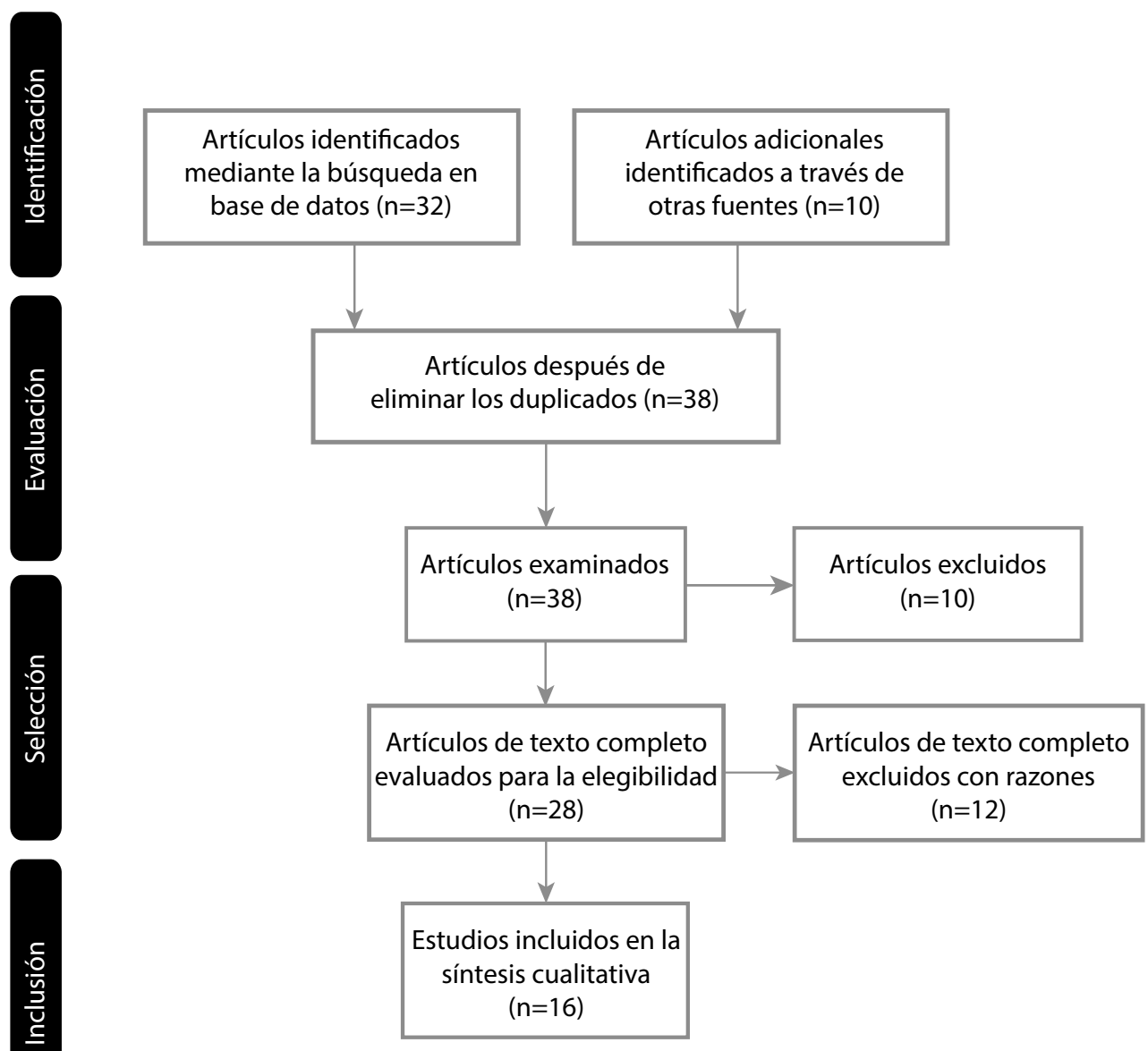

Figura 2: PRISMA. Diagrama de flujo. Elaboración propia.

\section{Resultados}

Eficacia, inmunogenicidad, persistencia de anticuerpos y seguridad

La eficacia de los estudios se valoró según la prevalencia de casos de los distintos tipos de VPH después de la aplicación de la vacuna. La inmunogenicidad demuestra la formación de anticuerpos para los diferentes tipos de VPH. Por otro lado, la persistencia de anticuerpos nos muestra si en los estudios se valoró la presencia de los mismos después de cierto tiempo (evaluado en cada estudio) y por cuánto tiempo se encontró efectividad de la vacuna.

La seguridad se evaluó con base en los efectos adversos; locales o sistémicos que llegaran a presentar o no los participantes de los estudios, donde los efectos locales más comunes son: dolor, inflamación y eritema en el sitio de punción (vacunación) de levemoderada intensidad. Los efectos sistémicos son: fiebre, náusea, vómito, mialgia, mareo y diarrea. Los efectos adversos más severos son la cefalea persistente, hipertensión, gastroenteritis y broncoespasmo. ${ }^{4}$ 


\section{Tabla 3}

\section{Estrategia de búsqueda.}

\begin{tabular}{|c|c|c|c|c|}
\hline $\begin{array}{l}\text { BASE DE } \\
\text { DATOS/ } \\
\text { BUSCADOR }\end{array}$ & PALABRAS CLAVE & $\begin{array}{l}\text { MARCADORES } \\
\text { BOOLEANOS }\end{array}$ & LIMITADORES & ARTículos \\
\hline PubMed & $\begin{array}{l}\text { "HPV", "cervical cancer", } \\
\text { "vaccination", } \\
\text { "Quadrivalent vaccine", } \\
\text { "nonavalent vaccine" }\end{array}$ & AND, OR & $\begin{array}{l}\text { Full text } \\
\text { Free full text }\end{array}$ & 8 \\
\hline $\begin{array}{l}\text { Science Direct } \\
\text { de Elsevier }\end{array}$ & $\begin{array}{l}\text { "HPV", "vaccination", } \\
\text { "Quadrivalent vaccine", } \\
\text { "nonavalent vaccine" }\end{array}$ & AND, OR & Full text & 10 \\
\hline $\begin{array}{l}\text { Google } \\
\text { Scholar }\end{array}$ & $\begin{array}{l}\text { "9HPVv" "HPV" "HPV } \\
\text { vaccination" "qHPVv" }\end{array}$ & AND, OR & Full text & 10 \\
\hline EBSCO & $\begin{array}{l}\text { "HPV", "vaccination", } \\
\text { "Quadrivalent vaccine", } \\
\text { "nonavalent vaccine" }\end{array}$ & AND, OR & Full text & 7 \\
\hline OXFORD & $\begin{array}{l}\text { "HPV", "vaccination", } \\
\text { "Quadrivalent vaccine", } \\
\text { "nonavalent vaccine" }\end{array}$ & AND, OR & $\begin{array}{l}\text { Full text } \\
\text { Free full text }\end{array}$ & 3 \\
\hline
\end{tabular}

La Tabla 4 muestra los resultados obtenidos en la evaluación de los artículos respecto a la eficacia profiláctica de la vacuna tetravalente y nonavalente en mujeres jóvenes. Las Tablas 5 y 6 muestran los resultados obtenidos en la evaluación de los artículos respecto a la eficacia, inmunogenicidad, persistencia de anticuerpos y seguridad de la vacuna nonavalente, vacuna tetravalente y placebo en los diferentes grupos de edad.

\section{Discusión}

Esta revisión sistemática representa más de 180 mil de personas de datos de seguimiento, los informes revelan que hay una disminución a nivel poblacional de los casos relacionados con el VPH después de la implementación de programas de vacunación contra este virus.

\section{Eficacia}

En la Tabla 4 se puede observar que la vacuna nonavalente muestra un mayor por- centaje de reducción de $\mathrm{VPH}$ respecto a la cuadrivalente; esto se puede determinar debido a que se compararon estudios que evaluaban la prevalencia de prevacunación contra la prevalencia postvacunación en cada una de las vacunas analizadas en el artículo (cuadrivalente y nonavalente), encontrando que, en el caso de la vacuna tetravalente, existía una prevalencia prevacunación en el artículo de Joura y cols. de $19.1 \%$ y en el Garland y cols. de $28.8 \%$ comparado con la postvacunación en la que se obtuvo una prevalencia de $6.5 \%$ y $16.7 \%$ respectivamente, observando una disminución de $66 \%$ y $42 \%$. Respecto a la vacuna nonavalente, se observó que existía una prevalencia prevacunación $28.7 \%$ según Pitisuttithum y cols. y de $22 \%$ según Garland y cols., y una prevalencia postvacunación de 6.5 y $6 \%$ respectivamente; teniendo una disminución de prevalencia de $78 \%$ y $73 \%$ respectivamente. 


\section{Tabla 4}

Prevalencia de VPH pre-vacunación y post-vacunación.

\begin{tabular}{|c|c|c|c|c|}
\hline Referencia & Población de estudio & $\begin{array}{c}\text { Prevalencia VPH } \\
\text { pre-vacunación }\end{array}$ & $\begin{array}{l}\text { Prevalencia VPH } \\
\text { post-vacunación }\end{array}$ & $\begin{array}{l}\text { Porcentaje de } \\
\text { reducción }\end{array}$ \\
\hline \multicolumn{5}{|l|}{ qvVPH } \\
\hline Joura et al. & Mujeres 16-26 años. & $19.1 \%$ & $6.5 \%$ & $66 \%$ \\
\hline Garland et al. & Mujeres de $15-26$ años. & $28.8 \%$ & $16.7 \%$ & $42 \%$ \\
\hline \multicolumn{5}{|l|}{$9 v V P H$} \\
\hline $\begin{array}{l}\text { Pitisuttithum } \\
\text { et al. }\end{array}$ & Mujeres 16-26 años. & $28.7 \%$ & $6.5 \%$ & $78 \%$ \\
\hline Garland et al. & Mujeres de $12-26$ años. & $22 \%$ & $6 \%$ & $73 \%$ \\
\hline
\end{tabular}

En la tabla 5, se puede observar las características de los estudios analizados y en la tabla 6, que en la mayoría de los ensayos clínicos hubo una mayor disminución de la prevalencia del VPH $(31,33,45,52,58)$ en las pacientes que fueron vacunadas con la vacuna nonavalente en comparación con aquellas a las que les fue aplicada la vacuna tetravalente. . $^{4-8}$

Todo esto representa una disminución estadísticamente significativa de las dos vacunas, siendo mucho menor la incidencia con la nonavalente, debido a que provee una protección mayor ya que cubre contra más tipos de VPH.

Se observa además que en aquellos ensayos en los que se comparan poblaciones de entre 16 y 26 años, con poblaciones de entre 9 y 16 años, la eficacia de la vacuna nonavalente en general tenía un efecto equiparable en ambos grupos de edad. 6,7,9 Sin embargo, en otros estudios, se observa mayor eficacia de la vacuna nonavalente en el grupo de edad de los 9 a los 16 años. ${ }^{10}$

Esto nos indica que, aunque la eficacia puede ser similar entre grupos de edad tan alejados, se debe optar por vacunarse en el rango de 9-16 años, para que si, aun cuando la eficacia sea igual, podría ser posible optimizarla en algunos casos.

Por otro lado, en estudios en los que se comparaba la eficacia de la vacuna tetravalente contra placebo, se observó una reducción significativa con la vacuna tetravalente en la aparición de verrugas genitales, ${ }^{11-13}$, y reducción de la prevalencia de cáncer cervical intraepitelial. ${ }^{14}$

La administración de la vacuna nonavalente tuvo una mayor eficacia en el grupo al que se le aplicó dicha vacuna, comparada con el grupo al que se le aplicó placebo con previa aplicación de la vacuna tetravalente. $^{15}$

En la figura 3 se observa, de forma esquematizada, los principales resultados de la revisión, respecto a la eficacia se puede observar que la vacuna nonavalente presenta una mayor eficacia clínica en los tipos de VPH - 31, 33, 45, 52 y 58 en comparación con la vacuna tetravalente. 


\section{Tabla 5}

Evaluación de las características de los estudios.

\begin{tabular}{|c|c|c|c|c|c|c|c|c|c|}
\hline Referencia & Diseño del estudio & $\begin{array}{c}\text { Ubicación del } \\
\text { estudio }\end{array}$ & Periodo de estudio & $\begin{array}{c}\text { Población de } \\
\text { estudio }\end{array}$ & $\begin{array}{l}\text { Tamaño de la } \\
\text { muestra }\end{array}$ & Seguimiento & Intervención & Comparación & $\begin{array}{l}\text { Resultados } \\
\text { evaluados }\end{array}$ \\
\hline Skinner y cols & $\begin{array}{c}\text { Fase III. Ensayo } \\
\text { clínico aleatorizado, } \\
\text { doble ciego. Estudio } \\
\text { VIVIANE }\end{array}$ & $\begin{array}{l}\text { Multicéntrico } 12 \\
\text { páses }\end{array}$ & $\begin{array}{l}\text { Febrero 2006-di- } \\
\text { ciembre } 2010\end{array}$ & Mujjeres $>25$ años & 5,752 & 40-43 meses & Vacuna tetravalente & $\begin{array}{l}\text { Vacuna contra tipos } \\
\text { no oncogénicos }\end{array}$ & $\begin{array}{l}\text { Eficacia (mujeres } \\
\text { adultas), seguridad, } \\
\text { inmunogenicidad }\end{array}$ \\
\hline Tota y cols & $\begin{array}{l}\text { Ensayo clínico alea- } \\
\text { torizado, doble ciego. } \\
\text { Estudio PATRICIA }\end{array}$ & Multicéntrico 4 países & $2004-2005$ & $\begin{array}{l}\text { Mujeres 15-25 años } \\
\text { y mujeres de } 18-25 \\
\text { años }\end{array}$ & 26,195 & 2 años & $\begin{array}{c}\text { Vacuna contra tipos } \\
\text { oncogénicos (dosis } \\
0,1 \text { y } 6 \text { meses) }\end{array}$ & $\begin{array}{c}\text { Vacuna contra tipos } \\
\text { no oncogénicos } \\
\text { (dosis } 0,1 \text { y } 6 \\
\text { meses) }\end{array}$ & Eficacia \\
\hline Petersen y cols & $\begin{array}{l}\text { Estudio clínico en } \\
\text { fase lll }\end{array}$ & Unicéntrico EUA & Sin especificar & $\begin{array}{c}\text { Mujeres y hombres } \\
\text { de } 9 \text { a } 15 \text { años y } \\
\text { mujeres de } 16-26 \\
\text { años }\end{array}$ & 11,304 & Sin especificar & $\begin{array}{l}\text { Vacuna nonavalente } \\
\text { en mujeres y hom- } \\
\text { bres de } 9 \text { a } 15 \text { años }\end{array}$ & $\begin{array}{c}\text { Vacuna nonavalente } \\
\text { en mujeres de } 16 \text { a } \\
26 \text { años }\end{array}$ & $\begin{array}{c}\text { Inmunogenicidad, } \\
\text { eficacia }\end{array}$ \\
\hline Muñoz y cols & $\begin{array}{l}\text { Estudio clínico con- } \\
\text { trolado aleatorizado, } \\
\text { placebo-controlado } \\
\text { en fase III }\end{array}$ & $\begin{array}{l}\text { Multicéntrico } 24 \\
\text { páses }\end{array}$ & $\begin{array}{l}\text { Diciembre 2001- } \\
\text { mayo } 2003\end{array}$ & Mujeres 15-26 años & 17,622 & $\begin{array}{l}\text { En promedio } 3.6 \\
\text { años, máximo } \\
4.9 \text { años }\end{array}$ & $\begin{array}{c}\text { Vacuna tetravalente } \\
\text { (dosis } 0,1,2 \text { y } 6 \\
\text { meses) }\end{array}$ & $\begin{array}{c}\text { Placebo (dosis 0, 1,2 } \\
\text { y6 meses) }\end{array}$ & Eficacia \\
\hline Garland y cols & $\begin{array}{c}\text { Estudio clínico con- } \\
\text { trolado aleatorizado, } \\
\text { placebo-controlado } \\
\text { en fase III }\end{array}$ & $\begin{array}{l}\text { Multicéntrico } 24 \\
\text { países }\end{array}$ & $\begin{array}{l}\text { Diciembre 2001- } \\
\text { mayo } 2003\end{array}$ & Mujeres $15-26$ años & 17,622 & En promedio 4 años & $\begin{array}{l}\text { Vacuna tetravalente } \\
\text { (dosis } 0,1,2 \text { y } 6 \\
\text { meses) }\end{array}$ & $\begin{array}{c}\text { Placebo (dosis 0, 1,2 } \\
\text { y } 6 \text { meses) }\end{array}$ & Eficacia \\
\hline Garland y cols & $\begin{array}{c}\text { Ensayo clínico } \\
\text { aleatorizado, doble } \\
\text { ciego, fase III }\end{array}$ & Sin especificar & $\begin{array}{c}\text { Enero 2002-marzo } \\
2003\end{array}$ & Mujeres $16-24$ años & 5,455 & 3 años & Vacuna tetravalente & Placebo & Eficacia \\
\hline Stemberg y cols & $\begin{array}{c}\text { Ensayo clínico } \\
\text { aleatorizado, doble } \\
\text { ciego }\end{array}$ & $\begin{array}{l}\text { Multicéntrico } 18 \\
\text { países }\end{array}$ & Sin especificar & \begin{tabular}{|c|} 
Mujeres $16-26$ años \\
y hombres de 9 a \\
15 años
\end{tabular} & 5,312 & Sin especificar & Vacuna nonavalente & Vacuna tetravalente & $\begin{array}{l}\text { Eficacia, inmunogeni- } \\
\text { cidad, seguridad }\end{array}$ \\
\hline Damme y cols & $\begin{array}{l}\text { Ensayo clínico } \\
\text { aleatorizado }\end{array}$ & Sin especificar & Sin especificar & Mujeres & Sin especificar & Sin especificar & Vacuna nonavalente & Vacuna tetravalente & Eficacia \\
\hline Vesikari y cols & $\begin{array}{c}\text { Estudio aleatorizado, } \\
\text { doble ciego, con- } \\
\text { trolado }\end{array}$ & $\begin{array}{l}\text { Multicéntrico } 24 \\
\text { países }\end{array}$ & Sin especificar & Niñas de 9 a 15 años & 600 & 7 meses & Vacuna tetravalente & Vacuna nonavalente & $\begin{array}{l}\text { Inmunogenicidad, } \\
\text { seguridad, } \\
\text { tolerabilidad }\end{array}$ \\
\hline Luxemburg y cols & $\begin{array}{l}\text { Ensayo clínico } \\
\text { fase }\|/\| \|\end{array}$ & Multicéntrico & 2007 & Mujeres 16 a 26 años & 45,000 & Sin especificar & Vacuna nonavalente & Vacuna tetravalente & $\begin{array}{c}\text { Eficacia inmunoge- } \\
\text { nicidad }\end{array}$ \\
\hline Castellsaguéa y cols & $\begin{array}{l}\text { Ensayo clínico } \\
\text { aleatorizado }\end{array}$ & 76 sitios en 17 países & Octubre 2012-agosto & $\begin{array}{l}\text { Mujeres } 16-26 \text { años y } \\
\text { hombres } 16-26 \text { años }\end{array}$ & 2,500 & Sin especificar & $\begin{array}{c}\text { Vacuna nonavalente } \\
\text { en mujeres }\end{array}$ & $\begin{array}{c}\text { Vacuna nonavalente } \\
\text { en hombres }\end{array}$ & $\begin{array}{c}\text { Inmunogenicidad, } \\
\text { seguridad }\end{array}$ \\
\hline
\end{tabular}

\section{Inmunogenicidad}

En la Tabla 6 se observa que en aquellos pacientes a los que se les aplicó la vacuna nonavalente generaron anticuerpos contra VPH $(31,33,45,52,58)$, evento que no se observa en aquellos a los que se les aplicó la vacuna tetravalente. Además, se muestra que la generación de anticuerpos contra VPH (6, $11,16,18)$ fue equiparable tanto en aquellos pacientes a los que se le administró la vacuna nonavalente como en aquellos a los que se les administró la vacuna tetravalente, ${ }^{4-8,16}$ tal como se muestra en la figura 3.

La inmunogenicidad de la vacuna nonavalente en el estudio de Van Damme y cols. demostró que la generación de anticuerpos en ambos grupos de edad, 9 a 15 años y 16 a 26 años, fue equiparable. ${ }^{17}$ Por otro lado, Petersen y cols. demuestra que la respuesta fue mayor en el grupo de pacientes de 9 a 15 años que en el grupo de 16 a 26 años. 


\section{Tabla 6}

Resultados evaluados en los diferentes estudios.

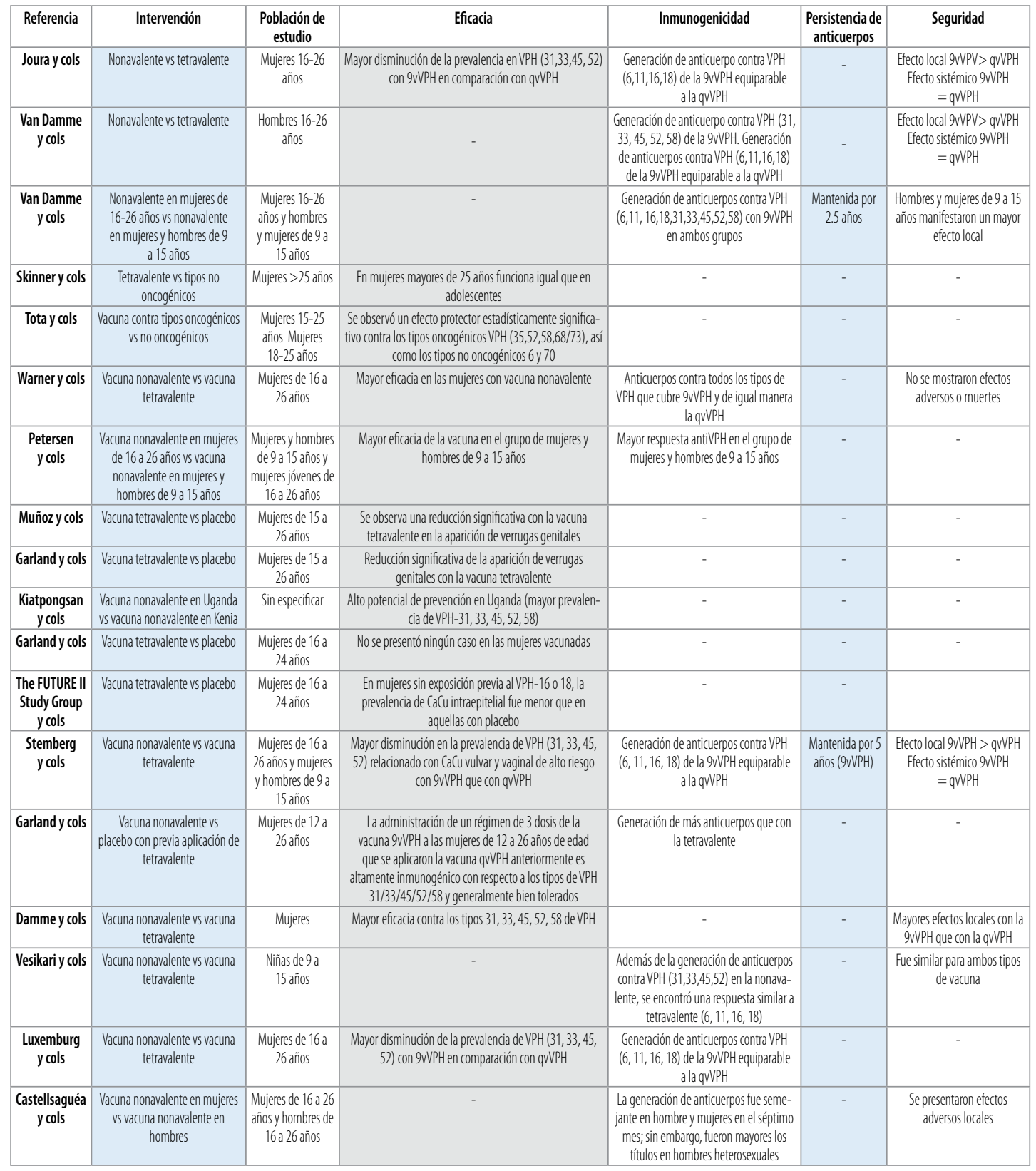

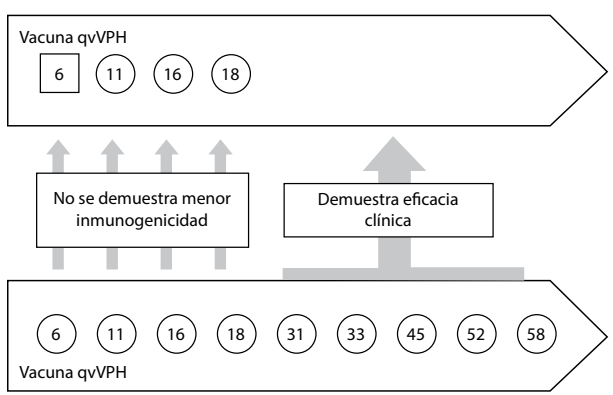

Figura 3: Principales resultados de la revisión 


\section{Persistencia de anticuerpos}

Un solo artículo reporta el tiempo de efectividad de la vacuna reflejada en la persistencia de anticuerpos después de cierto tiempo ${ }^{7}$, tal como lo muestra la tabla 6 , donde en las mujeres de 16 a 26 años y hombres y mujeres de 9 a 15 años, se mantuvo por 2.5 años.

\section{Seguridad}

Se destaca que aunque la eficacia de la vacuna sea del $100 \%$, la población toma la decisión de no vacunar a las niñas o los mismos adultos pueden no aplicarse la vacuna por miedo a la seguridad de la misma.
En la Tabla 6 se mencionan los efectos adversos tanto locales como sistémicos de las vacunas nonavalente y tetravalente, destacando en todos la incidencia de mayores efectos adversos locales con la aplicación de la vacuna nonavalente, mientras que los efectos sistémicos se presentaron de igual manera en ambas vacunas; sin embargo, la ocurrencia de estos efectos es relativamente baja.

También se puede valorar la seguridad con base en los datos mundiales de la aplicación de la vacuna, donde la presencia de efectos adversos se limita mayoritariamente a efectos locales.

\section{Conclusiones}

Los resultados de los ensayos clínicos aleatorizados realizados en mujeres jóvenes han permitido establecer el marco de seguridad, eficacia e inmunogenicidad de las vacunas tetravalente y nonavalente para el VPH. Además, han demostrado que ambas vacunas son altamente eficaces en la prevención de los tipos relacionados con los VPH de alto riesgo incluidos en la vacuna (VPHs 16 y 18); adicionalmente, la vacuna nonavalente muestra eficacia clínica contra los tipos 31, 33, 45, 52 y 58. Cabe destacar que ambas son vacunas profilácticas y no tienen ninguna eficacia terapéutica.

La vacuna tetravalente es eficaz en la prevención de verrugas genitales relacionadas con los VPH 6 y 11. Así mismo, la vacuna nonavalente tiene la capacidad de prevenir el cáncer cervical en un $70-90 \%$ y en un $85-95 \%$ la presentación de cáncer anal, vaginal y vulvar.

Hay que resaltar que, a pesar de los resultados obtenidos, se requiere continuar estudiando para dar respuesta a preguntas aún pendientes de resolver; como: ¿por cuánto tiempo la protección inducida por la primo-vacunación se mantendrá, o si son necesarias más dosis?; y de ser así, el esquema se puede cambiar para favorecer el aumento de la cobertura y reducir los costos; o preguntas en relación al perfil de seguridad, ya que el desarrollo de vacunas contra el VPH y la implementación de la vacunación en poblaciones preadolescentes pueden ser el principio en la erradicación del cáncer cervicouterino y de una drástica disminución de los cánceres de vagina, vulva, orofaringe, ano y pene. 


\section{Limitaciones}

Las limitaciones de este artículo recaen principalmente en los posibles sesgos que pueden existir debido a la heterogeneidad de las poblaciones y a que las características de las pacientes no son homogéneas en cuanto a los grupos de edad.

\section{Bibliografía}

1. Ochoa F, Guarneros D, Velasco J. Infección por virus del papiloma humano en mujeres y su prevención.

SMeO. 2015; 14 (3):157-163.

2. Barra F, Roberti U, Boganni G, Diotto A, Signorelli $M$, Chiappa V, Loruso D, Raspagliesi F, Ferrero S. New prophylactis human papiloma virus (HPV) vaccines against cervical cancer. Journal of Obstetrics and Gynaecology. (2018), https://doi.org/10.1080/01443 615.2018.1493441

3. Vanegas V, Rubio A, Milena A, Sánchez G. Estructura molecular y antigénica de la vacuna contra el virus del papiloma humano 16 (VPH 16). Acta biol. Colomb. 2008; 13(3):37-48.

4. Joura EA, Giuliano $A R$, Iversen $O E$, Bouchard $C$, Mao C, Mehlsen J, Moreira ED, et al. A 9-valent HPV vaccine against infection and intraepithelial neoplasia in women. N Engl J Med. 2015; 372(8):711-23.

5. Huh WK, Joura EA, Giuliano AR, Iversen $O E$, Andrade RP, Ault KA, Bartholomew $D$, et. al. Final efficacy, immunogenicity, and safety analyses of nine-valent human papillomavirus vaccine in women aged 16-26 years: a randomised, dule-blind trial. Lancet. 2017; 390:2143-59.

6.-Ruiz-Sternberg AM, Moreira ED, Restrepo JA, Lazcano-Ponce $E$, Cabello $R$, Silva $A$, Andrade $R$, et. al. Efficacy, immunogenicity, and safety of a 9-valent human paillomavirus vaccine in Latin American girls, boys, and young women. Papillomavirus Research. 2018; 5:53-74.

7. Van Damme $P$, Bonanni $P$, Bosch FX, Joura, E, Kjaer, SK, Meijer CJLM, Petry KU, et. al. Use of the nonavalent HPV vaccine in individuals previously fully or partially vaccinated with bivalent or quadrivalent HPV vaccines. Vaccine. 2016; 34:757-761.

8. Luxembourg A, Bautista $O$, Erin $M$, Ritter $M$, Chen J. Design of a large outcome trial for a multivalent human papillomavirus L1 virus-like particle vaccine. Contemporary Clinical Trials. 2015; 42:18-25.

9. Tota JE, Struyf $F$, Merikukka $M$, Gonzalez $P$, Kreimer $A R, B i D$, Castellsagué $X$, et. al. Evaluation of Type Replacement Following HPV16/18 Vaccination: Pooles Analysis of Two Randomized Trials. JNCI Natl Canc Inst. 2017; 109(7):djw300.

10. Peters LK, Rstpo J, Mra ED, Iveen OE, Ptisuttihum $P$, Van Damme P, Joura EA, et. al. Impact of baseline covariates on the immunogenicity of the 9-valent

\section{Conflictos de interés}

Los autores declaran no tener ningún conflicto de interés.

Los autores del presente trabajo no recibieron ningún patrocinio para llevarlo a cabo.

HPV vacine - A combined analysis of five phase III clinical trials. Papillomavirus Researh. 2017; 3:105115.

11. Muñoz N, Kjaer SK, Sigurdsson K, Iversen OE, Hernandez-Avila $M$, Wheeler $C M$, Perez $G$, et. al. Impact of Human Papillomavirus (HPV)-6/11/16/18 Vaccine on All HPV-Associated Genital Diseasses in Young Women. JNCl. 2010; 102(5):325-339.

12. Garland $S M$, Steben $M$, Sings $H L$, James $M$, Lu $S$, Raikar R, Barr E, et. al. Natural History of Genital Warts; Analysis of the Placebo Arm of 2 Randomized Phase III Trials of a Quadrivalent Human Papillomavirus (Types 6, 11, 16, and 18) Vaccine. JID. 2009; 199:805-814.

13. Garland $S M$, Hernandez-Avila $M$, Wheeler $C M$, Perez G, Harper DM, Leodolter S, Tang GWK, et. al.

Quadrivalent Vaccine Against Human Papillomavirus to Prevent Anogenital Diseases.N Engl J Med. 2007; 365(19): 1928-1943.

14. The FUTURE II Study Group. Quadrivalent Vaccine against Human Papillomavirus to Prevent High-Grade Cervical Lesions. N Engl J Med. 2007; 356(19):1915-1927.

15. Garland SM, Cheung TH, McNeill S, Petersen LK, Romaguera J, Vazquez-Narvez J, Bautista O, y cols. Safety and immunogenicity of a 9-valent HPV vaccine in females 12-26 years of age who previously received the quadrivalent HPV vaccine. Vaccine. (2015), http://dx.doi.org/10.1016/j.vaccine.2015.08.059.

16. Vesikari T, Brodszki N, Van Damme P, Diez-Domingo J, Icardi G, Petersen LK, Tran C, y cols. A Randomized, Double-Blind, Phase III Study of the Immunogenicity and Safety of a 9-Valent Human Papillomavirus L1 Virus-Like Particle Vaccine (V503) Versus Gardasil in 9-15 Year-Old Girls. Pediatr Infect Dis J. 2015; 34: 992-998.

17. Van Damme $P$, Olsson $S E$, Block $S$, Castellsague $X$, Gray GE, Herrera $T$, Huang LM, y cols. Immunogenicity and Safety of a 9-Valent HPV Vaccine. Pediatrics. 2015; 136(1):e28-e39.

18. Bruni L, Serrano B, Bosch $X$, Castellsagué $X$. Vacuna frente al virus del papiloma humano. Eficacia y seguridad. Enferm Infecc Microbiol Clin. 2015. http:// dx.doi.org/10.1016/j.eimc2015.03.018

19. Pitisuttihum $P$, Velicer $C$, Luxembourg A. 9-Valent HPV vaccine for cancers, pre-cancers and genital warts related to HPV. Expert Rev Vaccines. 2015; 1-15. 